\title{
Estimation of nonlinear systems parameters
}

\author{
M. Benyassi ${ }^{1}$, A. Brouri ${ }^{2}$ \\ ${ }^{1}$ Department of Electrical Engineering, LEM2A, ESTM, Moulay Ismail University, Morocco \\ ${ }^{2}$ AEEE Department, L2MC, IMSM, ENSAM, Moulay Ismail University, Morocco
}

\begin{tabular}{|c|c|}
\hline Article Info & ABSTRACT \\
\hline Article history: & In this paper, an identification method is proposed to determine the nonlinear \\
\hline Received Apr 23, 2019 & $\begin{array}{l}\text { systems parameters. The proposed nonlinear systems can be described by } \\
\text { Wiener systems. This structure of models consists of series of linear dynamic }\end{array}$ \\
\hline Revised Jul 5, 2019 & element and a nonlinearity block. Both the linear and nonlinear parts are \\
\hline Accepted Oct 6, 2019 & $\begin{array}{l}\text { nonparametric. In particular, the linear subsystem of structure entirely } \\
\text { unknown. The considered nonlinearity function is of hard type. This latter }\end{array}$ \\
\hline Keywords: & $\begin{array}{l}\text { can have a dead zone or with preload. These nonlinear systems have been } \\
\text { confirmed by several practical applications. The suggested approach involves }\end{array}$ \\
\hline Frequency system identification & \\
\hline
\end{tabular}

Hard nonlinearity

Nonlinear system identification

Nonlinear Wiener models

Nonparametric linear block

This is an open access article under the CC BY-SA license.

\section{Corresponding Author:}

Mohamed Benyassi,

Department of Electrical Engineering ESTM,

Moulay Ismail University,

Route d'Agouray, km 5, B.P. 3103, Toulal, Meknès, 50000, Morocco.

Email: benyassimohamed@yahoo.fr

\section{INTRODUCTION}

The Wiener model is a series connection of a linear dynamic bloc and a memoryless nonlinearity (Figure 1). Nonlinear systems structured in block are widely studied in the last decades [1-10]. When both parts are parametric, the identification problem has been dealt with using stochastic methods, e.g. [1, 2] as well as deterministic methods, e.g. [11, 12]. The stochastic methods enjoy local or global convergence properties under various assumptions e.g. the system inputs should be persistently exciting (PE) or Gaussian and the system nonlinearity is invertible. The last limitation has recently been overcome in [12]. Multi-stage methods, involving two or several stages, have been proposed in e.g. [13, 14] and their consistency was ensured if the inputs are Gaussian and the nonlinearity is odd. Deterministic parameter identification methods consist in reformulating the problem as an optimization task, generally coped with using various relaxation techniques. Then, local convergence properties ensured in presence of PE inputs. In Nonparametric Wiener systems (where none of the linear subsystem or the nonlinear element assumes a priori known structure) have been dealt with using both stochastic and frequency methods. In the stochastic methods e.g. [15], the nonlinearity is generally determined using variants of the kernel regression estimation technique while the (unknown) coefficients of a FIR/IIR approximation of the linear part are estimated using cross-correlation analysis. Several assumptions are needed e.g. Gaussian inputs, FIR linear dynamics, Lipschitzian nonlinearity. In frequency methods, the linear subsystem frequency response and the nonlinearity map are determined in two or several stages, e.g. $[16,17]$. The frequency identification of nonlinear system has been widely dealt by Brouri for several structures and models [16-19]. Presently, the considered nonlinear system is characterized by nonparametric linear block and can be of unknown structure. Furthermore, the system nonlinearity is of hard type (Figure 2). This latter may have several phenomena; e.g. saturation, preload, dead 
zone. Presently, the system nonlinearity considered in Wiener models is very difficult to be modeled or approximated by polynomial function or any other basis functions.

The aim in this work is to develop an identification method allowing to estimate the hard system nonlinearity, as well as an accurate estimate of linear block parameters. The proposed identification approach can be developed in two stages. In the first stage, using simple constant inputs an accurate estimate of the nonlinearity functions $\mathrm{f}($.$) can be given. In the second stage, the frequency modulus gain |G(j \omega)|$ and the argument (the phase) $\angle G(j \omega)$ of $G(j \omega)$, for a set of frequencies $\omega \in\left\{\omega_{1}, \omega_{2}, \ldots, \omega_{m}\right\}$.

The outline of the remaining part of this paper consists of 3 sections. The system nonlinearity identification is coped with in Section 2. The determination of the linear subsystem frequency response is investigated in Section 3. Section 4 is devoted to the conclusion.

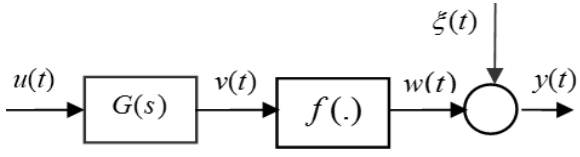

Figure 1. Wiener Model structure

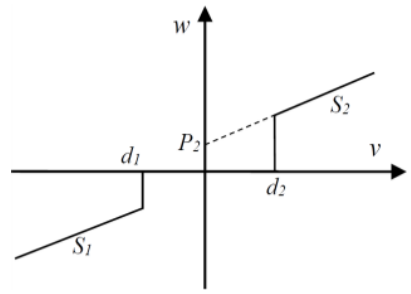

Figure 2. Nonlinearity hard with preload and dead zone

\section{IDENTIFICATION OF SYSTEM NONLINEARITY}

Standard Wiener models consist of a linear element of transfer function $G(s)$ followed in series by a static nonlinear function $f($.$) . The Wiener systems considered in this work is characterized by set of$ internal signals $v(t)$ and $w(t)$, and $\xi(t)$ (Figure 1), which are inaccessible to measurement. The external signals $u(t)$ and $y(t)$ respectively denote the control input and the measured output. The inner signal $\xi(t)$ accounts for measurement noise and other modeling effects. It is supposed to be zero-mean ergodic and uncorrelated with the control input $u(t)$. Accordingly, let $g(t)$ denotes the impulse response of the linear block of transfer function $G(s)$. equations:

More specifically, the Wiener system under study is analytically described by the following

$$
\begin{aligned}
& v(t)=g(t) * u(t) \\
& w(t)=f(v(t)) \\
& y(t)=w(t)+\xi(t)
\end{aligned}
$$

where $g(t)=L^{-1}(G(s))$ and $L^{-1}$ designates the inverse Laplace transform. The symbol $*$ in $(1)$ refers to the convolution product.

Presently, the considered linear block can be of unknown structure, but it is just supposed to be asymptotically stable. This assumption makes possible open-loop system identification. In this section, an identification schema is proposed to estimate the hard system nonlinearity (Figure 2). Then, the Wiener system is excited by a set of constant inputs:

$$
u(t) \in\left\{U_{j} ; j=1 \ldots n\right\}
$$

where the number of input excitations $\mathrm{n}$ in (1) is arbitrarily chosen by the user.

In this problem identification, the complexity also lies in the fact that the internal signal $v(t)$ is not uniquely defined from an input-output viewpoint (see e.g. [16-19]). Then, without loss of generality, one can assume that the linear block to be identified is characterized by unit static gain:

$$
G(0)=1
$$

Indeed, if the couple $(G(s), f()$.$) is solution of this nonlinear identification problem (1)-(3), then any$ nonlinear system having the following linear subsystem block: 


$$
G^{*}(s)=\frac{G(s)}{\lambda}
$$

and the following system linearity:

$$
f^{*}(v)=f(\lambda v)
$$

is also solution of the above nonlinear identification problem (1)-(3), where is any nonzero real number. Accordingly, the judicious choice of the distribution factor in the present paper is:

$$
\lambda=G(0)
$$

Finally, one immediately gets from (6a) and (6c) that, the linear block $G^{*}(s)$ to be identified is of unit static gain:

$$
G^{*}(0)=1
$$

On the other hand, it is readily seen using (1) and (4)-(5) that:

$$
v(t) \in\left\{U_{j} ; j=1 \ldots n\right\}
$$

Equation (7) means that, for any constant input $U_{j}$ the steady state of inner signal $v(t)$ is also constant and converges towards $U_{j}$ (i.e. of known values). Accordingly, one can immediately gets from (2) and (7) that the undisturbed system output $w(t)$ converges towards set constant values. Specifically, one has:

$$
w(t) \in\left\{f\left(U_{j}\right) ; j=1 \ldots n\right\}
$$

Finally, it follows from (3) and (8) that, for any input $U_{j}$, the system output $y(t)$ converges to a constant up to noise:

$$
y(t) \in\left\{f\left(U_{j}\right)+\xi(t) ; j=1 \ldots n\right\}
$$

Then, using the fact that, the extra-input (noise) signal $\xi(t)$ is zero-mean, an accurate estimate of $f\left(U_{j}\right)$ (for $j=1 \ldots n$ ) can be obtained by averaging the system output $y(t)$. To this end, let $\widehat{W}_{j}(j=1 \ldots n)$ denotes the estimate of $w(t)$ for $u(t)=U_{j}$. Then, the estimator of $w(t)$ for any $j \in\{1, \ldots, n\}$ is as follows:

$$
\widehat{W}_{j}=\frac{1}{N} \sum_{t=1}^{N} y(t)
$$

Accordingly, it readily follows from (3), (9) and (10) that:

$$
\widehat{W}_{j}=f\left(U_{j}\right)+\frac{1}{N} \sum_{t=1}^{N} \xi(t)
$$

One can see that the last term in (11) vanishes with probability equal to 1 . Accordingly, the estimate $\widehat{W}_{j}$ can be expressed as (w.p.1): that:

$$
\widehat{W}_{j} \approx f\left(U_{j}\right) \text { for } j=1 \ldots n
$$

For more information, see e.g. [16-19]. Accordingly, a set of points belonging to nonlinearity function $f($. can be determined.

\section{RESEARCH METHOD}

The aim in this section is to develop an identification solution allowing estimates of linear block parameters. Presently, the proposed Wiener model is characterized by nonparametric linear element. This latter can be of unknown structure. Accordingly, the objective of this identification method provides 
the frequency complex gain of linear subsystem, i.e. for any frequency $\omega \in\left\{\omega_{1}, \ldots \omega_{m}\right\}$ we seek an estimate of the modulus gain $|G(j \omega)|$ and the phase $\varphi(\omega)=\angle G(j \omega)=\arg (G(j \omega))$.

To do this, the Wiener model (Figure 1 ) is excited by a sine input signal:

$$
u(t)=u_{0}+U \cos (\omega t), \text { with } \omega \in\left\{\omega_{1}, \ldots \omega_{m}\right\}
$$

Then, it follows from (1), (5) and (13) that, the steady state of inner signal $\mathrm{v}(\mathrm{t})$ is also sine signal and can be expressed as follows:

$$
v(t)=u_{0}+U|G(j \omega)| \cos (\omega t+\varphi(\omega))
$$

On the other hand, recalling that the system nonlinearity $f($.$) is of hard function and is already determined$ (Section 2). Then, the choice of $u_{0}$ and $U$ can be done such that the signal $v(t)$ spans only one segment of $f($.$) . In that case, the steady-state of undisturbed system output w(t)$ is also sine signal. Specifically, $\mathrm{w}(\mathrm{t})$ can be expressed as:

$$
w(t)=u_{0}+b+a U|G(j \omega)| \cos (\omega t+\varphi(\omega))
$$

where $\mathrm{a}$ and $\mathrm{b}$ are the segment parameters.

A judicious choice of $u_{0}$ and $U$ can be made practically by observing the system output $y(t)$. Then, adjusting the offset and the amplitude until $y(t)$ becomes close to a sinusoidal signal up to noise. Accordingly, it readily follows from the (3) and (15) that, the system output $y(t)$ can be expressed as:

$$
y(t)=u_{0}+b+a U|G(j \omega)| \cos (\omega t+\varphi(\omega))+\xi(t)
$$

Furthermore, the question in this stage is how to estimate the inner signal $w(t)$ using input and output system? The answer to this question can be found in [20]. Know that the input signal $u(t)$ and the system output $y(t)$ are $T$ - periodic (of same period T). Then, an accurate estimate $\widehat{w}(t)$ of $w(t)$ can be obtained by trans-averaging $y(t)$ (see [20]):

$$
\widehat{w}(t)=\frac{1}{M} \sum_{l=1}^{M} y(t+l T)
$$

Furthermore, it follows from (16) and (17) that, the output estimate $\widehat{w}(t)$ can be expressed as:

$$
\widehat{w}(t)=u_{0}+b+a U|G(j \omega)| \cos (\omega t+\varphi(\omega))+\frac{1}{M} \sum_{l=1}^{M} \xi(t+l T)
$$

Bear in mind that, the signal $\xi(t)$ is a white noise process with zero mean. Then, the expression of $\widehat{w}(t)$ in (18) can be reduced to the following equation as:

$$
\widehat{w}(t)=u_{0}+b+a U|G(j \omega)| \cos (\omega t+\varphi(\omega))
$$

Equation (19) shows that the estimate $\widehat{w}(t)$ of internal signal $w(t)$ converges to its true value (w.p.1). Specifically, one has:

$$
\widehat{w}(t)=w(t) \quad \text { w.p. } 1
$$

Let $\hat{S}(\omega)$ and $\hat{\alpha}(\omega)$ respectively denote the amplitude and phase of the sinusoidal term of $\widehat{w}(t)$. Then, the frequency gain estimate (modulus gain $|\hat{G}(j \omega)|$ and phase $\hat{\varphi}(\omega)=\angle \hat{G}(j \omega)$ ) can be given as:

$$
\begin{aligned}
& |\hat{G}(j \omega)|=\frac{\hat{S}(\omega)}{a U} \\
& \hat{\varphi}(\omega)=\hat{\alpha}(\omega) \quad(\text { modulo } 2 \pi)
\end{aligned}
$$

This experience can be repeated for any other frequency $\omega \in\left\{\omega_{1}, \ldots \omega_{m}\right\}$. 


\section{SIMULATION}

The Wiener systems considered in simulation is described linear subsystem of transfer function:

$$
G(s)=\frac{0.1}{(s+0.1)(s+0.4)}
$$

Then, the system nonlinearity $f($.$) considered in simulation shown by Figure 3$. Accordingly, to overcome the multiplicity of solutions problem seen in section 2 , we have shown that it is judicious to focus to nonlinear system described by $(6 a-c)$. Then, the nonlinear system to be identified is analytically described by linear element of following transfer function:

$$
G^{*}(s)=\frac{0.04}{(s+0.1)(s+0.4)}
$$

Furthermore, it is readily seen from (23) that the linear element of Wiener nonlinear system to be identified satisfies the property $(5)$, i.e. $G^{*}(s)$ is of unit static gain $\left(G^{*}(0)=1\right)$. In the first stage, the Wiener system is excited by a set of constant inputs (Figure 4$)$. The resulting system output $y(t)$ and the undisturbed output estimates $\widehat{W}_{j}$ are shown by Figure 5 . For convenience, the system nonlinearity $f($.$) and the set of$ estimated points $\left(U_{j}, \widehat{W}_{j}\right)(j=1 \ldots 9)$ are plotted in Figure 6. Finally, the obtained simulation values and given in Table 1 shown that the estimated points are very close to their true values.

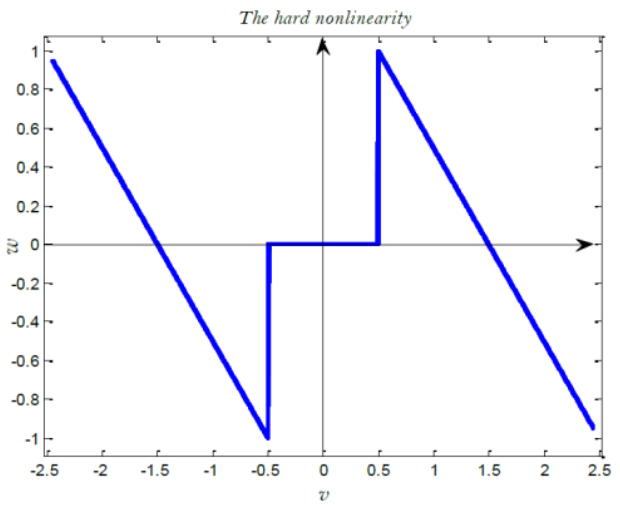

Figure 3. The nonlinearity considered in simulation

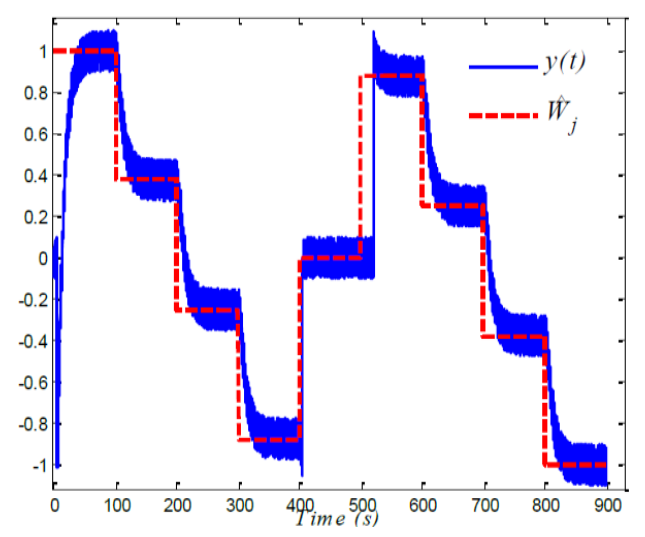

Figure 5. The output $y(t)$ and the estimates $\widehat{W}_{j}$

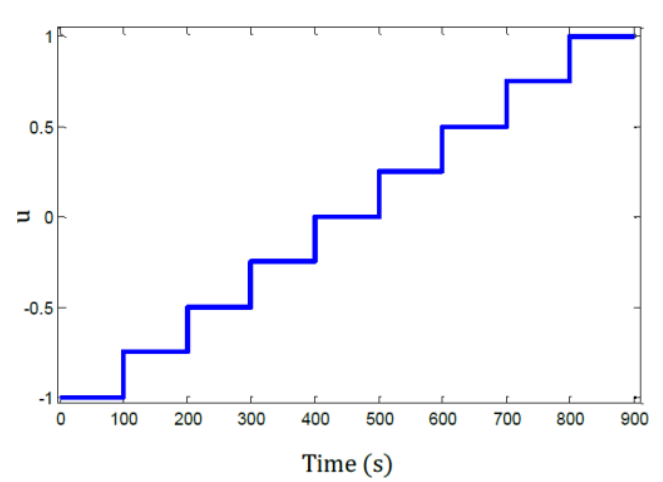

Figure 4. The input signal $u(t)$

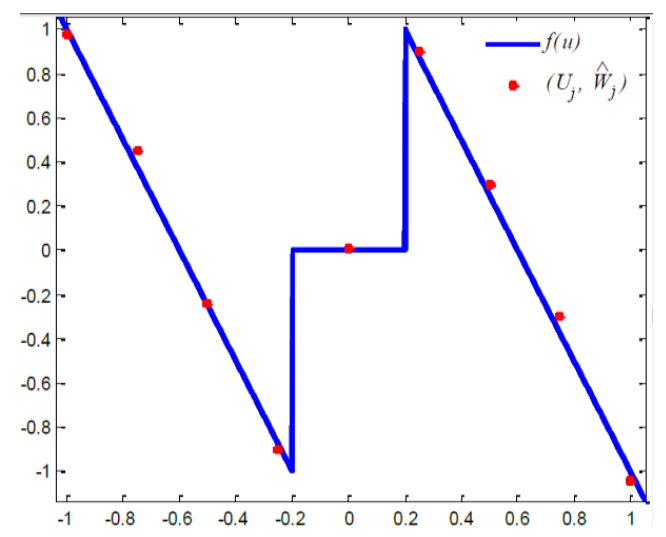

Figure 6. The NL $f($.$) and the estimates \left(U_{j}, \widehat{W}_{j}\right)$

Table 1. Nonlinear points estimates $\hat{f}\left(U_{j}\right)$

\begin{tabular}{cccc}
\hline $\mathrm{J}$ & 4 & 5 & 7 \\
\hline$U_{j}$ & -0.25 & 0 & 0,5 \\
$f\left(U_{j}\right)$ & -0.87 & 0 & 0,25 \\
\hline
\end{tabular}




\begin{tabular}{llll}
$\hat{f}\left(U_{j}\right)$ & -0.88 & 0.02 & 0,27 \\
\hline
\end{tabular}

In the second stage (see section 3), the Wiener nonlinear system is excited by the sine signal (13). For an arbitrarily offset $u_{0}$ and amplitude $U$, the system output $y(t)$ over one period is shown by Figure 7 . Then, it is clear that the signal $y(t)$ is different from a sinusoidal signal.

Accordingly, by adjusting the values of $u_{0}$ and $U$ in (13) and using the nonlinearity estimate. Furthermore, using the system nonlinearity estimate (Figure 6), useful values for $u_{0}$ and $U$ in (13) can be easily done. Then, another example for $u_{0}=0.25$ and $U=0.5$ is displayed by Figure 8 .

This result showed that the system output is not yet a sine signal. Indeed, the filtered version using the estimator (17) is presented in Figure 9. It is seen that the signal $y(t)$ is different from a sine signal. Then, adjusting the parameters of input signal $u(t)$ (i.e. the values of offset $u_{0}$ and amplitude $U$ ) until the system output $y(t)$ becomes sine signal (up to noise). The observed system output $y(t)$ in this case is illustrated by Figure 10.

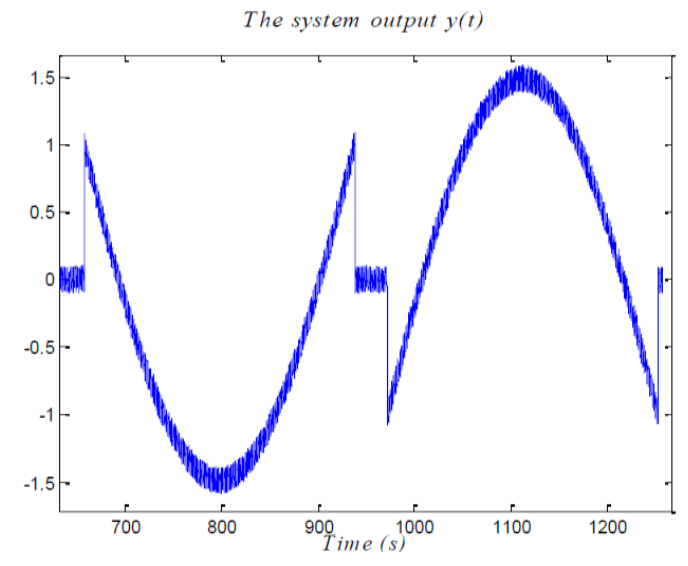

Figure 7. The system output $y(t)$ over one period

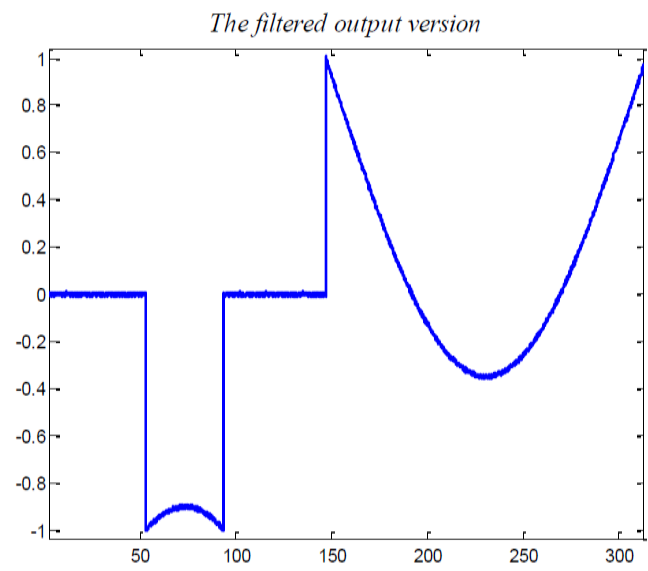

Figure 9. The filtered output $\widehat{w}(t)$ over one period

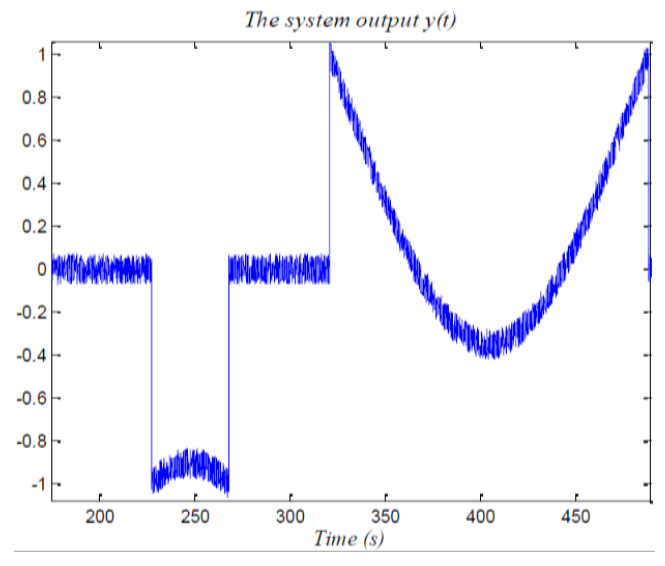

Figure 8. The output $y(t)$ over one period

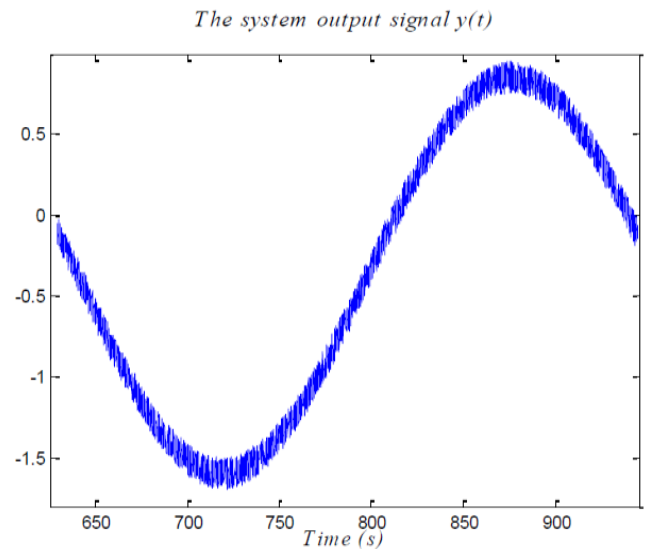

Figure 10. The output $y(t)$ over one period

This result shows that the signal $y(t)$ is close to a sine signal (up to noise). The collected sample of $y(t)$ is used to generate the undisturbed output estimate $\widehat{w}(t)$ using the estimator (17). This latter is plotted by Figure 11 . It is remarkable that $\widehat{w}(t)$ is close to a sine signal. Then, for the frequency $\omega=0.01(\mathrm{rd} / \mathrm{s})$, the obtained amplitude and phase of $\widehat{w}(t)$ are as follows:

$$
\begin{aligned}
& \hat{S}(\omega)=1.01 \\
& \hat{\alpha}(\omega)=3.04(r d)
\end{aligned}
$$


Then, based upon the system nonlinearity identification, the frequency complex gain (modulus gain $|\widehat{G}(j \omega)|$ and phase $\hat{\varphi}(\omega)=\angle \hat{G}(j \omega)$ ) of linear block can be given using (21a-b).

Bear in mind that $\hat{a} \approx-0.99$, then it readily follows from $(21 a-b)$ and (24a-b) that, the parameters estimate (modulus gain and phase) of frequency complex gain for $\omega=0.01(\mathrm{rd} / \mathrm{s})$ are (respectively):

$$
\begin{aligned}
& |\hat{G}(j \omega)|=\frac{\hat{S}(\omega)}{a U}=1.01 \\
& \hat{\alpha}(\omega)=3.04-\pi \approx-0.1(r d)
\end{aligned}
$$

Repeat this method for other frequencies $\omega \in\left\{\omega_{1}, \ldots \omega_{m}\right\} . s$

It follows (13a-b) and using the amplitude and phase of $\widehat{w}(t)$, can be obtained for any frequency $\omega$. The obtained estimate values of the modulus gain $|\hat{G}(j \omega)|$ and phase $\hat{\varphi}(\omega)$ or three frequencies $\omega \in$ $\left\{\omega_{1}, \ldots \omega_{m}\right\}$ are given in Table 2. Finally, the obtained results given in Table 2 show that, the complex gain parameters estimate are very close to their true values.

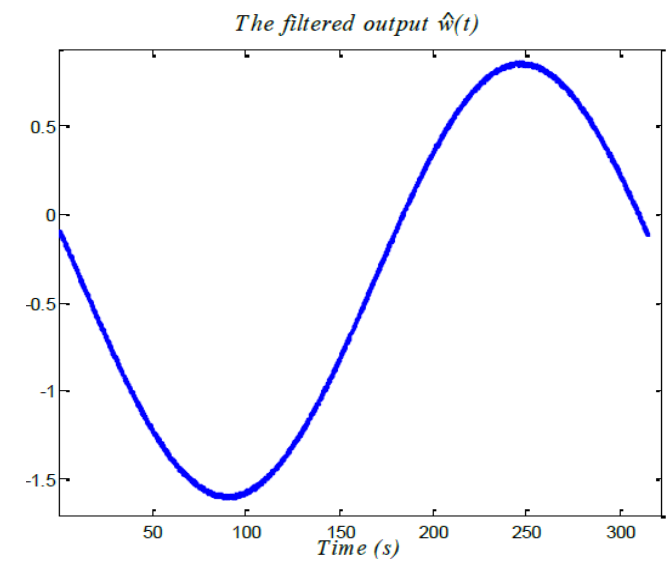

Table 2. Frequency gain estimates $\hat{G}\left(j \omega_{k}\right)$

\begin{tabular}{cccc}
\hline$k$ & 1 & 2 & 3 \\
\hline$\omega_{k}(\mathrm{rd} / \mathrm{s})$ & 0.01 & 0.05 & 0.1 \\
$\varphi\left(\omega_{k}\right)(\mathrm{rd})$ & -0.12 & -0.59 & -1.03 \\
$\hat{\varphi}\left(\omega_{k}\right)(\mathrm{rd})$ & -0.1 & -0.62 & -1.06 \\
$\left|G\left(j \omega_{k}\right)\right|$ & 0.99 & 0.89 & 0.68 \\
$\left|\hat{G}\left(j \omega_{k}\right)\right|$ & 1.01 & 0.87 & 0.71 \\
\hline
\end{tabular}

Figure 11. The undisturbed output estimate $\widehat{w}(t)$ over one period

\section{CONCLUSION}

In this paper, a new frequency identification method is proposed to deal with Wiener model. Presently, the identification problem is addressed in presence of hard nonlinearity and linear dynamic element of unknown structure. In the first stage, an identification method is presented to estimate the hard nonlinearity. Then, a frequency-domain identification solution is developed allowing the determination of frequency complex gain (i.e. modulus gains estimate, as well as the phase) estimate of linear block.Presently, the linear element is nonparametric and can be of unknown structure. Furthermore, the system nonlinearity can be discontinuous.

\section{REFERENCES}

[1] T. Wigren, "Recursive prediction error identification using the nonlinear Wiener model," Automatica, vol. 29, pp. 1011-1025, 1993.

[2] T. Wigren, "Convergence analysis of recursive identification algorithm based on the nonlinear Wiener model," IEEE Trans. Automatic Control, vol. 39. pp. 2191-2205, 1994.

[3] A. Brouri, F. Giri, "Modeling of nonlinear systems with backlash memory operators," in 10th International Conference on Modeling, Optimization and Simulation (MOSIM'14), Nancy, France, November 5-7, 2014.

[4] A. Brouri, "Frequency Identification of Wiener-Hammerstein Systems," in SIAM Conference on Control \& Its Applications, Paris, France, July 8-10, 2015.

[5] A. Brouri, "Frequency Identification Of Nonlinear Systems," in 18th International Conference on Automatic Control, Modelling \& Simulation, Venice, Italy, January 29-31, 2016.

[6] A. Wills and L. Ljung, Wiener system identification using the maximum likelihood method. In Block-oriented nonlinear system identification, U.K.: Springer, 2010. 
[7] L. Vanbeylen, R. Pintelon, and J. Schoukens, "Blind maximum-likelihood identification of wiener systems," IEEE Transactions on Signal Processing, vol. 57, no. 8, pp. 3017-3029, 2009.

[8] L. Vanbeylen and R. Pintelon, Blind maximum-likelihood Identification of Wiener and Hammerstein nonlinear block structures. Block-oriented nonlinear system identification, U.K.: Springer, 2010, pp 273-291.

[9] A. Brouri, "Wiener-Hammerstein Models Identification," Int. Journal of Math. Mod. \& Meth. in Applied Sc., vol. 10, pp. 244-250, 2016.

[10] A. Brouri, "Nonlinear System Identification," in International Conference on Applied Mathematics \& Computer Science (ICAMCS), Rome, Italy, January 27-29, 2017.

[11] J. Vörös, "Parameter identification of Wiener systems with discontinuous nonlinearities," Systems and Control Letters, vol. 44, pp. 363-372, 1997.

[12] J. Bruls, C.T. Chou, B.R.J. Heverkamp and M. Verhaegen, "Linear and nonlinear system identification using separable least squares," European Journal of Control, vol. 5, pp. 116-128, 1999.

[13] D. Westwick and M. Verhaegen, "Identifying MIMO Wiener systems using subspace model identification methods," Signal Processing, 52, pp. 235-258, 1996.

[14] M. Lovera, T. Gustafsson, and M. Verhaegen, "Recursive subspace identification of linear and non-linear Wiener state-space models," Automatica, vol. 36, pp. 1639-1650, 2000.

[15] W. Greblicki and M. Pawlak, Nonparametric System Identification. Cambridge University Press, 2008.

[16] A. Brouri, F. Giri, F.Z. Chaoui, A. Amdouri, "Identification of Hammerstein-Wiener Systems with Backlask Input Nonlinearity Bordered By Straight Lines," in 19th IFAC World Congress, Cape Town, South Africa, August 24-29, 2014, pp. 475-480.

[17] A. Brouri, L. Kadi, S. Slassi, "Frequency identification of Hammerstein-Wiener systems with Backlash input nonlinearity," Int. J. of Control, Automation \& Systems, vol. 15, no. 5, pp. 2222-2232, 2017.

[18] A. Brouri, F.Z. Chaoui, A. Amdouri, F. Giri, "Frequency Identification of Hammerstein-Wiener Systems with Piecewise Affine Input Nonlinearity," in 19th IFAC World Congress, Cape Town, South Africa, August 24-29, 2014, pp. 10030-10035.

[19] A. Brouri, S. Slassi, "Identification of Nonlinear Systems Structured by Wiener-Hammerstein Model", International Journal of Electrical and Computer Engineering, vol. 6, no. 1, pp. 167-176, 2016.

[20] A. Brouri, Frequency Identification of Nonlinear Systems, LAP LAMBERT Academic Publishing, September, 2016, ISBN: 978-3- 659-94991-3.

\section{BIOGRAPHIES OF AUTHORS}

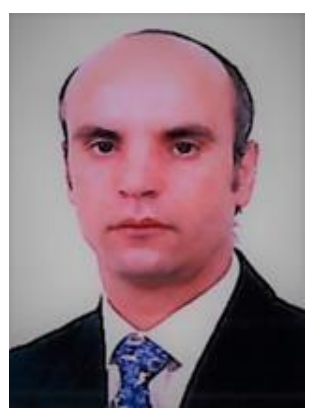

Mohamed Benyassi has completed his Master degree in Physics and Mathematics from Faculty of Science and Mathematics Russian University Moscow USSR in 1991. In 1995 he received the $\mathrm{Ph}$. D. degree in theoretical Physics from the same University. Since 2004 he joined the High School of Technology ESTM, Moulay Ismail University in Meknes Morocco. Prof. Benyassi joined the LEM2A Lab as a permanent member in Moulay Ismail University. His research interests nonlinear and inverse systems. E-mail: benyassimohamed@yahoo.fr

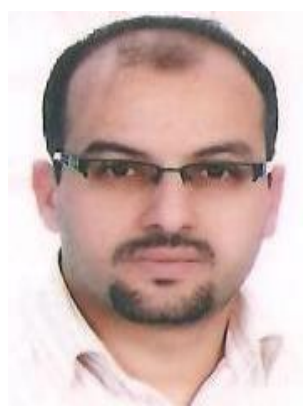

Adil Brouri obtained the Agrégation of Electrical Engineering in 2000. In 2012, he obtained a Ph.D. in Automatic Control from the University of Mohammed 5, Morocco. He has been Professeur- Agrégé for several years. Since 2013 he joined the ENSAM, My Ismail University in Meknes- Morocco. He obtained his HDR degree in 2015 at the ENSAM-Meknes (My Ismail University). His research interests include nonlinear system identification and nonlinear control. He published several papers on these topics. Prof. Brouri joined the research team "IMSM" and the L2MC Laboratory as a permanent member. E-mail: a.brouri@ensam-umi.ac.ma \& brouri_adil@yahoo.fr 